\title{
Size tunable visible and near-infrared photoluminescence from vertically etched silicon quantum dots
}

\author{
Sameer S. Walavalkar, ${ }^{1,2, a)}$ Andrew P. Homyk, ${ }^{1,2}$ Carrie E. Hofmann, ${ }^{2}$ M. David Henry, ${ }^{1,2}$ \\ Claudia Shin, ${ }^{2}$ Harry A. Atwater, ${ }^{2}$ and Axel Scherer ${ }^{2}$ \\ ${ }^{1}$ Kavli Nanoscience Institute, Caltech, Pasadena California 91125, USA \\ ${ }^{2}$ Department of Applied Physics, Caltech, Pasadena California 91125, USA
}

(Received 31 January 2011; accepted 31 March 2011; published online 14 April 2011)

\begin{abstract}
Corrugated etching techniques were used to fabricate size-tunable silicon quantum dots that luminesce under photoexcitation, tunable over the visible and near infrared. By using the fidelity of lithographic patterning and strain limited, self-terminating oxidation, uniform arrays of pillar containing stacked quantum dots as small as $2 \mathrm{~nm}$ were patterned. Furthermore, an array of pillars, with multiple similar sized quantum dots on each pillar, was fabricated and tested. The photoluminescence displayed a multiple, closely peaked emission spectra corresponding to quantum dots with a narrow size distribution. Similar structures can provide quantum confinement effects for future nanophotonic and nanoelectronic devices. (c) 2011 American Institute of Physics.
\end{abstract}

[doi:10.1063/1.3580768]

Over the past 60 years silicon devices have evolved to form the backbone of the micro-electronics industry. Unfortunately, the indirect nature of the silicon band-gap has hampered the material with highly inefficient light emission properties, thus impeding its spread into the optoelectronic field. Recent work, however, has focused on utilizing the physical changes seen in low dimensional nanostructures to breathe life into the use of silicon as an active optical material.

Silicon quantum wells, ${ }^{1}$ grown nanowires, ${ }^{2}$ etched nanopillars, ${ }^{3}$ and quantum dots ${ }^{4,5}$ have shown promising electroluminescent and photoluminescent properties. Specifically silicon nanocrystals embedded in a dielectric matrix such as silicon dioxide or silicon nitride have been used as a convenient method to generate complimentary metal on semiconductor (CMOS) compatible layers of light emitting material. The most popular technique to fabricate these nanocrystals has been to deposit a layer of silicon rich oxide or nitride and anneal at above $1100{ }^{\circ} \mathrm{C}$, allowing the excess silicon to precipitate into clusters within the dielectric. ${ }^{6-8}$ Depending on the annealing temperature, a statistical distribution of quantum dot sizes is found. It has been shown that these dots can be made to luminesce by optical pumping as well as through electrical excitation by tunneling electrons and holes through the dielectric and allowing them to recombine in the dots.

Although this precipitation technique has been improved and well characterized over the years, it comes with some key limitations. For example, the spatial distribution of these dots cannot be controlled accurately which makes addressing individual quantum dots difficult. Furthermore, the inherent size distribution of the precipitated nanoclusters leads to inhomogeneous broadening and artificially widened emission spectra. $^{7}$

Recent work ${ }^{9}$ has shown a remarkable effort into the fabrication and characterization of single etched quantum dots. These top-down devices have demonstrated narrow linewidths and 'blinking' behavior associated with emission

${ }^{a)}$ Electronic mail: walavalk@caltech.edu. from single nanocrystals. ${ }^{9-11}$ In this letter we demonstrate the ability to fabricate uniform arrays of stacked pillars of silicon quantum dots whose size (and thus peak emission wavelength) can be precisely tuned via etching parameters and oxidation conditions. These vertical quantum dots exhibit bright photoluminescence (PL) from the visible to the near infrared and can be predictably and repeatably placed through lithographic techniques.

Aluminum oxide disks to mask vertical quantum dots were patterned following previous techniques. ${ }^{3}$ The samples were etched using a "pseudo-Bosch" recipe using $\mathrm{SF}_{6}$ and $\mathrm{C}_{4} \mathrm{~F}_{8}$ to simultaneously etch and passivate, respectively. Under a fixed gas ratio and forward power the etch produces pillars with vertical sidewalls at an etch rate of roughly 250 $\mathrm{nm} / \mathrm{min} ;{ }^{3}$ an example of this etch can been seen in frame (b) of Fig. 1. By tuning the gas ratio as the etch progresses into the silicon we controllably undercut the etch mask to generate structures with arbitrarily corrugated features as small as $30 \mathrm{~nm}$; an example of this etch technique can been seen in

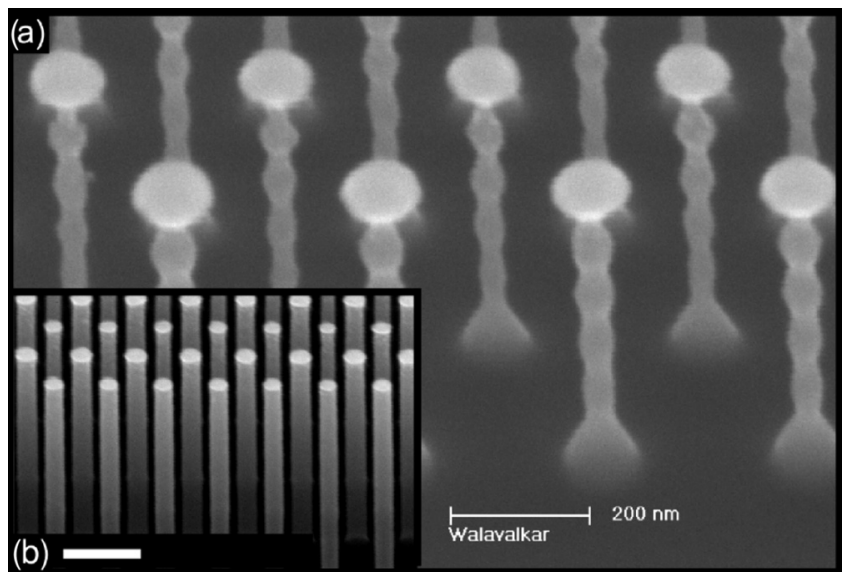

FIG. 1. (a) SEM image of an array of corrugated silicon nanopillars immediately after etching. These pillars were fabricated by oscillating the etching conditions to controllably undercut and overpassivate the silicon. (b) An array of nanopillars with vertical sidewalls etched using the same pseudoBosch recipe but without varying the ratio of etch to passivation gas. 


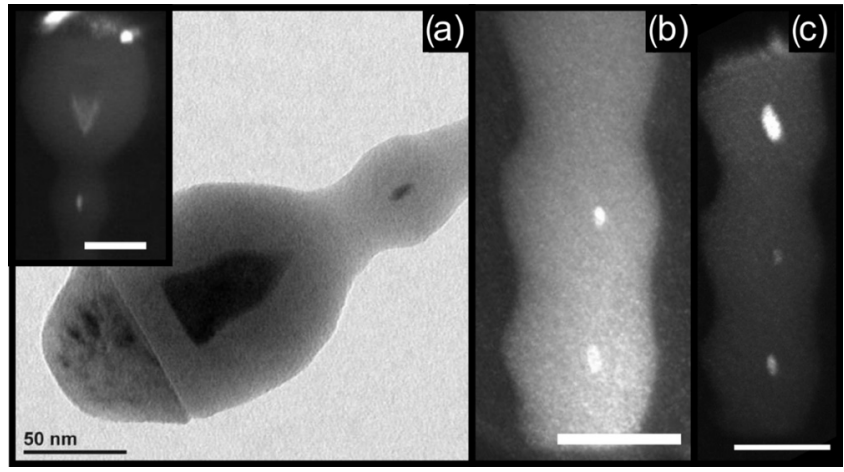

FIG. 2. (a) Bright-field image of the head and first quantum dot of a corrugated pillar after oxidation. Inset shows a similar picture with diffraction contrast to highlight the crystalline nature of the remaining silicon nanocrystals. [(b) and (c)] These frames utilize diffraction contrast to highlight the remaining quantum dots in the legs of the corrugated pillars after selfterminating oxidation. Scale bars are $50 \mathrm{~nm}$.

Fig. 1(a). In order to prevent additional undercut as the etch continues we increased the forward power to better collimate the ions.

The second step in the fabrication is the self-terminating oxidation. It has been shown ${ }^{2,3,12}$ that when convex silicon structures are oxidized at temperatures below $950{ }^{\circ} \mathrm{C}$, the oxidation self-terminates predictably leaving a silicon core encapsulated by silicon dioxide. The oxidation process ends when the compressive strain at the $\mathrm{Si}-\mathrm{SiO}_{2}$ interface prevents oxygen diffusion to the oxidation front. By selecting the oxidation temperature as well as the initial dimensions of the convex silicon structure the size of the remaining silicon core can be tuned. ${ }^{3,11}$

Samples were fabricated with original mask diameters of 80 and $100 \mathrm{~nm}$ and were undercut to produce three uniform corrugations per pillar. The corrugation size was varied between samples. The samples were simultaneously oxidized at $915^{\circ} \mathrm{C}$ for seven hours and were cooled to room temperature in a forming gas of 20:1 nitrogen and hydrogen. The presence of an elliptical silicon quantum dot was confirmed nondestructively by reflection mode transmission electron microscopy (TEM) (Ref. 3) as well as destructively after PL testing by transferring the quantum dots onto a TEM grid and viewing the structures in transmission mode.

Figure 2 shows TEM images of vertical quantum dots after oxidation. The first image shows the morphology of the surface after oxidation as well as a bright-field image of the silicon core in the head and first quantum dot of the pillar. The inset shows a similar view in dark-field with diffraction contrast used to highlight the single crystal nature of the remnant quantum dot. Frames (b) and (c) of Fig. 2 show the presence of crystalline silicon within the oxidized cores of the legs of the corrugated nanopillars.

The three samples tested are shown before oxidation as colored frames in Fig. 3, the diameters are roughly (a) $30 \mathrm{~nm}$ (black), (b) $37 \mathrm{~nm}$ (blue), and (c) $45 \mathrm{~nm}$ (green) and the corrugations have a period of approximately $60 \mathrm{~nm}$ for each sample. Micro-PL was performed in an inverted optical microscope setup with a $457 \mathrm{~nm}$ free-space argon ion laser used to pump the sample; the full experimental setup is described in previous work. ${ }^{3}$ The data collected from three samples of different preoxidation size is shown in Fig. 3. The color of the frame around the scanning electron microscope (SEM) images corresponds to the curve plotted in the figure. It can

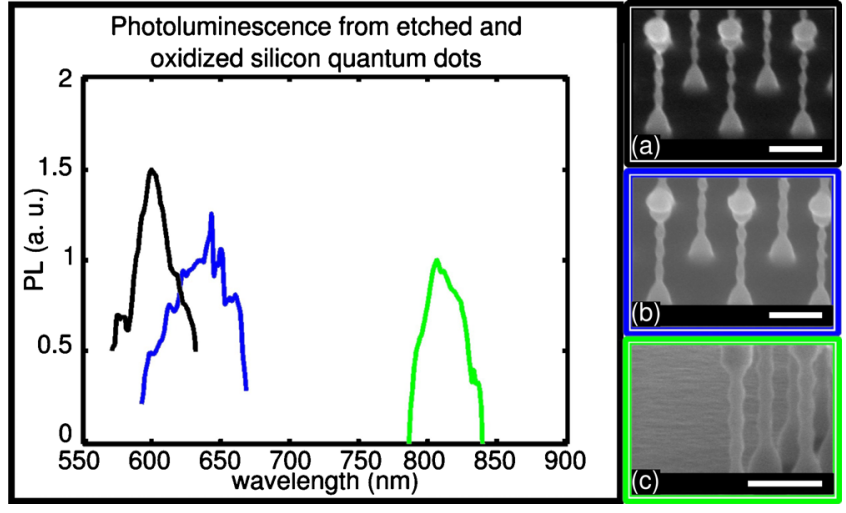

FIG. 3. (Color online) PL spectra of three samples of etched and oxidized quantum dots with different initial corrugation diameters. The leftmost curve (centered at $600 \mathrm{~nm}$ ) corresponds to pillars in frame (a), the middle curve (centered at $640 \mathrm{~nm}$ ) corresponds to the pillars in frame (b), and the rightmost curve (centered at $810 \mathrm{~nm}$ ) corresponds to the pillars in frame (c). The preoxidation size is $30 \mathrm{~nm}, 37 \mathrm{~nm}$, and $45 \mathrm{~nm}$ for the (a) black, (b) blue, and (c) green samples, respectively. Note that the larger the preoxidation size of the corrugated pillars the longer the peak emission wavelength. Scale bars are $200 \mathrm{~nm}$ in each frame.

be seen that there is a correlation between the original size of the etched corrugations and the peak emission wavelength of the oxidized quantum dot. Peak emission was found to be at roughly $600 \mathrm{~nm}(2.06 \mathrm{eV}), 640 \mathrm{~nm}(1.94 \mathrm{eV})$, and $810 \mathrm{~nm}$ $(1.53 \mathrm{eV})$, for the samples with initial diameters of $30 \mathrm{~nm}$ (black), $37 \mathrm{~nm}$ (blue), and $45 \mathrm{~nm}$ (green), respectively, with a full width half maximum of $150 \mathrm{meV}$ or less for each sample.

Although careful effort was made to produce quantum dots with diameters that had as narrow a size distribution as possible, the peak emission wavelength is a strong function of dot size $e^{8,13}$ and even a change in diameter of $0.25 \mathrm{~nm}$ (about one monolayer of $\mathrm{Si}$ ) can shift the peak emission energy up to $100 \mathrm{meV}(30 \mathrm{~nm}){ }^{8}$ Several causes, including noncircular mask patterning, debris on the wafer, and local etch variation could have such an impact on the peak emission wavelength. Furthermore, it has been shown ${ }^{2,3}$ that the strain incorporated into thermally oxidized silicon nanostructures can have a significant impact on the bandgap, shifting the peak emission energy by $200 \mathrm{meV}$ between $1 \%$ compressive and tensile strain. The assumption made in this work is that the three stacked quantum dots have the same size and strain conditions; however this is not necessarily true, especially when it is considered that the top and bottom quantum dots are capped with a head and tail, while the central quantum dot is bracketed by two other dots. These two causes can possibly account for the multiply-peaked structure of the three measured emission spectra. This explanation is consistent with the observations that the individual peaks, which make up the total curves, have widths that fall within or close to the previously measured ${ }^{10} 75-100 \mathrm{meV}(20-30 \mathrm{~nm})$ linewidths of room temperature, etched quantum dots.

The sharp peaks seen in the blue curve in Fig. 3 can be attributed to such a cause or to a similar effect combined with a limitation of the experiment. Converting the number of fabricated pillars per pad into an areal density yields a value of $\sim 5 \times 10^{9} \mathrm{~cm}^{-2}$; roughly three orders of magnitude less dense than coalesced nanoparticles. ${ }^{4}$ In our case, the $50 \times$ objective we used allowed sampling a $5 \times 5 \mu \mathrm{m}^{2}$ area corresponding to between 300 and 500 pillars, leading to a 
relatively discrete spectrum where each pillar could significantly contribute to the total signal. The relatively low collected signal from the few pillars in combination with the preferential scattering of the light from certain quantum dot pillars could give those pillars an unequal contribution to the signal; resulting in such peaks. Future work is needed to quantitatively explain these spectral details; performing measurements at cryogenic temperatures could narrow the linewidths and allow the emission from individual quantum dots to be assessed. Similar effects, resulting in multiply and sharply-peaked spectra, have been seen in previous work. ${ }^{6,9}$

From the dark and light-field TEM images it is possible to estimate the size of the quantum dots in the oxidized pillar; however the distortion found in each image due to the image being taken through about 50 to $75 \mathrm{~nm}$ of silicon dioxide makes the bounds of error too large for a meaningful size measurement. Instead, dot sizes were determined by comparing the peak emission energy with the band-gaps obtained by previous theoretical and experimental work. ${ }^{8,14}$ It is also critical to note that these dots are embedded in an oxide matrix which has been found ${ }^{13,14}$ to redshift the peak emission by almost $1 \mathrm{eV}$ compared to bare quantum dots or those with a hydrogen terminated surface. Based on data presented in Refs. 8, 10, 13, and 14 we estimate that the measured nanocrystal sizes are centered around $2 \mathrm{~nm}$ (black), $2.4 \mathrm{~nm}$ (blue), and $5 \mathrm{~nm}$ (green) in diameter.

The ability to fabricate silicon quantum dots with predictable sizes could prove to be useful when attempting to incorporate them with existing silicon photonic structures such as waveguides or photonic crystals. Instead of using a stochastic distribution of nanocrystals, these can be placed lithographically to coincide with the peak of the optical mode. The ability to tailor the peak emission of the quantum dots allows one to select the emission wavelength to suit a task or create a vertical stack of different dot sizes to allow for broad spectral emission. Furthermore, there has been interest in producing transistors with quantum dots that demonstrate quantum effects and Coulomb blockade at noncryogenic temperature. To retain quantum behavior at room temperature the quantum dot must be aligned between two electrodes and smaller than $5 \mathrm{~nm}$ in diameter. ${ }^{15}$ By turning the fabrication vertical, structures similar to those described in this paper could overcome the challenge of patterning gates with difficult, lateral electron beam lithography.
In conclusion, we have presented a CMOS compatible method to fabricate narrow band, luminescent silicon quantum dots. By controlling the size during lithography and etching, it is possible to utilize the self-terminating nature of convex silicon oxidation to predictably tune the peak emission wavelength. We have also demonstrated the ability to stack quantum dots of various predictable sizes in order to tailor the spectral behavior of these corrugated pillars. These devices and behaviors may have important applications in both future nanophotonic and nanoelectronics devices.

S. Walavalkar would like to thank Erika Garcia as well as Professor Tom Tombrello and Ryan Briggs for useful discussion. We would also like to gratefully acknowledge the Boeing corporation under the CT-BA-GTA-1 grant, the Advanced Energy Consortium under the BEG10-07 grant, and DARPA for generous support under the NACHOS (Grant No. W911NF-07-1-0277) program. A. Homyk would like to thank the ARCS foundation for their support. M. D. Henry would like to thank the John and Fannie Hertz Foundation for their funding.

${ }^{1}$ K. D. Hirschman, Proceedings of the UGIM Symposium Microelectronics Education for the Future Twelfth Biennial University/Government/ Industry Microelectronics Symposium, 1997, Vol. 177.

${ }^{2}$ A. R. Guichard, D. N. Barsic, S. Sharma, T. I. Kamins, and M. L. Brongersma, Nano Lett. 6, 2140 (2006).

${ }^{3}$ S. S. Walavalkar, C. E. Hofmann, A. P. Homyk, M. D. Henry, H. A. Atwater, and A. Scherer, Nano Lett. 10, 4423 (2010).

${ }^{4}$ X. Wen, L. V. Dao, and P. Hannaford, J. Phys. D: Appl. Phys. 40, 3573 (2007).

${ }^{5}$ R. J. Walters, G. I. Bourianoff, and H. A. Atwater, Nature (London) 4, 143 (2005).

${ }^{6}$ T.-Y. Kim, N.-M. Park, K.-H. Kim, G. Y. Sung, Y.-W. Ok, T.-Y. Seong, and C.-J. Choi, Appl. Phys. Lett. 85, 5355 (2004).

${ }^{7}$ L. Pavesi, L. Dal Negro, C. Mazzoleni, G. Franzò, and F. Priolo, Nature (London) 408, 440 (2000).

${ }^{8}$ M. Wolkin, J. Jorne, P. Fauchet, G. Allan, and C. Delerue, Phys. Rev. Lett. 82, 197 (1999).

${ }^{9}$ J. Valenta, R. Juhasz, and J. Linnros, Appl. Phys. Lett. 80, 1070 (2002).

${ }^{10}$ I. Sychugov, R. Juhasz, J. Valenta, and J. Linnros, Phys. Rev. Lett. 94, 087405 (2005).

${ }^{11}$ B. Bruhn, F. Sangghaleh, and J. Linnros, Phys. Status Solidi A 208, 631 (2010)

${ }^{12}$ H. I. Liu, D. K. Biegelsen, F. A. Ponce, N. M. Johnson, and R. F. W. Pease, Appl. Phys. Lett. 64, 1383 (1994).

${ }^{13}$ J. Wilcoxon, G. Samara, and P. Provencio, Phys. Rev. B 60, 2704 (1999).

${ }^{14}$ C. Garoufalis, A. Zdetsis, and S. Grimme, Phys. Rev. Lett. 87, 276402 (2001).

${ }^{15}$ E. Leobandung, L. Guo, Y. Wang, and S. Y. Chou, Appl. Phys. Lett. 67, 938 (1995). 